\title{
Use of Control Bar Matrix for Outbreak Detection in Syndromic Surveillance System
}

\author{
Tao Tao', Qi Zhao', Huijian Cheng², Lars Palm³ ${ }^{3}$ Xin Lu' ${ }^{4}$, Hui Yuan², Xiaoxiao Song ${ }^{1}$ and \\ Biao Xu*1 \\ ${ }^{1}$ School of Public Health, Fudan University, Shanghai, China; ${ }^{2}$ Jiangxi Provincial Center for Disease Control and Prevention, \\ Nanchang, China; ${ }^{3}$ Future Position X, Gävle, Sweden; ${ }^{4}$ Division of Global Health (IHCAR), Department of Public Health Sciences, \\ Karolinska Institutet, Stockholm, Sweden; ${ }^{5}$ College of Information Systems and Management, National University of Defense \\ Technology, Changsha, China
}

\section{Objective}

To develop and test the method of incorporating different control bars for outbreak detection in syndromic surveillance system.

\section{Introduction}

Aberration detection methods are essential for analyzing and interpreting large quantity of nonspecific real-time data collected in syndromic surveillance system. However, the challenge lies in distinguishing true outbreak signals from a large amount of false alarm (1). The joint use of surveillance algorithms might be helpful to guide the decision making towards uncertain warning signals.

\section{Methods}

A syndromic surveillance project (ISSC) has been implemented in rural Jiangxi Province of China since August 2011. Doctors in the healthcare surveillance units of ISSC used an internet-based electronic system to collect information of daily outpatients, which included 10 infectious related symptoms. From ISSC database, we extracted data of fever patients reported from one township hospital in GZ town between August 1st and December 31st, 2011 to conduct an exploratory study. Six different control bar algorithms, which included Shewart, Moving Average (MA), Exponentially Weighted Moving Average (EWMA) and EARS' C1, C2, C3, were prospectively run among historical time series of daily fever count to simulate a real-time outbreak detection. Each control bar used 7 days' moving baseline with a lag of 2 days [the baseline for predicting Day(t) starts from Day(t-9) to Day(t-3), C1 method used a lag of zero day]. We set the threshold of $\mu+2 \sigma$ for Shewart and MA, and 2.1 for EWMA C1, C2 and C3. An alarm was triggered when the observed data exceeded threshold, and the detailed information of each patient were checked for signal verification. Microsoft Excel 2007 was used to calculate the simulation results.

\section{Results}

During the 5 months, GZ township hospital reported 514 outpatients with fever symptom, with an average of 3.4 per day. All control bars were simultaneously operated among daily counts of fever cases. Of the 153 days on surveillance, 29 triggered alarms by at least one of the control bars. Nine days triggered alarms from $>=3$ control bars while on one day (12/30) all 6 algorithms raised alarms. Figure 1 shows the date, fever count, algorithm and warning level (color) of each alarm, which we called a control bar matrix. It can be seen that C3 and EWMA present a higher sensitiveness towards tiny data change whereas $\mathrm{C} 1, \mathrm{C} 2$ and MA focus on large increase of data. $\mathrm{C} 3$ also had a memory effect on recent alarms. No infectious disease epidemic or outbreak event was confirmed within the signals. Most fever patients on the nine high-warning days (red and purple) were diagnosed as upper level respiratory infection. However, we discovered that the sharp increase of fever cases on $12 / 30$ was attributed to $5 \mathrm{du}-$ plicate records mistakenly input by the staff in GZ hospital.

\section{Conclusions}

By combining control bars with different characteristics, the matrix has potential ability to improve the specificity of detection while maintaining a certain degree of sensitivity. With alarms categorized into hierarchical warning levels, public health staffs can decide which alarm to investigate according to the required sensitivity of surveillance system and their own capacity of signal verification. Though we did not find any outbreak event in the study, the possibility of localized influenza epidemic on high-warning days cannot be wiped out, and the matrix's ability to detect abnormal data change was apparent. The proper combination, baseline and threshold of control bars will be further explored in the real-time surveillance situation of ISSC.

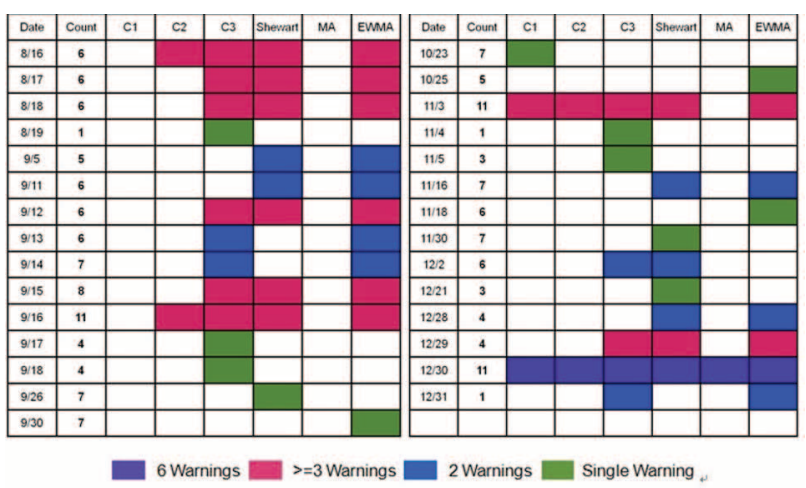

Figure 1: Detailed information of alarm signals generated by control bar matrix (No-alarm days were omitted)

\section{Keywords}

Syndromic surveillance; matrix; control bar; signal

\section{Acknowledgments}

This study was funded by [European Union's] [European Atomic Energy Community's] Seventh Framework Programme ([FP7/2007-2013] [FP7/2007-2011]) under grant agreement no. [241900].

\section{References}

1. Fearnley L: Signals come and go: syndromic surveillance and styles of biosecurity. Environment and Planning A 2008, 40(7):1615-1632.

*Biao Xu

E-mail: bxu@shmu.edu.cn 